\title{
Inmunodetección de metaloproteinasas de matriz extracelular (MMPs)-2, -9, -13 y -14 en lesiones apicales asociadas con periodontitis apical asintomática
}

\author{
Immunodetection of matrix metalloproteinases (MMPs)-2, -9, -13 and -14 in \\ periapical lesions associated with asymptomatic apical periodontitis
}

\author{
Mundi Burgos V1,5, Dezerega Piwonka A ${ }^{2,5}$, Osorio Alfaro C ${ }^{1,5}$, Dutzan Muñoz N ${ }^{3,5}$, Franco Martínez ME \\ Ortega Pinto AV ${ }^{4}$, Hernández Ríos M $M^{4,5}$
}

\begin{abstract}
RESUMEN
La periodontitis apical asintomática (PAa) es una patología infecciosa caracterizada por destrucción ósea perirradicular asociada a un proceso inflamatorio crónico y producción de mediadores inflamatorios, entre los cuales se encuentran las metaloproteinasas de matriz extracelular (MMPs). Entre éstas, las MMPs-13, -14, -2 y -9, son producidas por el tejido óseo y degradan sinérgicamente el colágeno tipo I, principal componente de los tejidos periodontales, y gelatina, producto de la degradación y desnaturación del colágeno. El objetivo de este estudio fue determinar el patrón de expresión de las MMPs-2, -9, -13 y -14 en granulomas periapicales (GPAs), quistes radiculares inflamatorios (QRIs) y ligamento periodontal sano (LS). Materiales y Métodos: Se seleccionaron 12 pacientes con diagnóstico clínico de PAa e indicación de exodoncia a partir de los cuales se obtuvieron biopsias de lesiones periapicales (LPAs). Como controles, se seleccionaron 7 individuos con indicación de exodoncia de premolares por ortodoncia, obteniéndose biopsias de LS. Se efectuó el diagnóstico anátomo-patológico de los especímenes y se caracterizó la expresión de las MMPs en estudio mediante inmunohistoquímica. Resultados: Las MMPs en estudio sólo se detectaron en GPAs y QRIs, y se inmunolocalizaron principalmente en el infiltrado inflamatorio de éstos. Adicionalmente, la MMP-2 se identificó en fibroblastos del tejido conectivo. Conclusiones: MMPs-2, -9 , -13 y -14 se expresan predominantemente en el infiltrado inflamatorio de las LPAs y no en LS, y por tanto se sugiere la participación de estos mediadores en la patogénesis de la PAa.
\end{abstract}

Rev. Clin. Periodoncia Implantol. Rehabil. Oral Vol. 4(1); 17-21, 2011.

Palabras clave: Periodontitis apical asintomática, lesiones periapicales, metaloproteinasas de matriz extracelular.

\begin{abstract}
Asymptomatic apical periodontitis (aAP) is an infectious disease characterized by perirradicular bone destruction associated with chronic inflammation and release of inflammatory mediators, such as matrix metalloproteinases (MMPs). MMPs-13, -14 and -2 , -9 are bone-expressed enzymes that can synergistically degrade collagen I, the main component of periodontal extracellular matrix, and gelatin, the product of degradation and denaturation of collagen. The aim of this study was to characterize the expression pattern of MMPs-2, $-9,-13$, and -14 in periapical granulomas (PGs), radicular cysts (RCs) and healthy periodontal ligament (PDL). Materials and Methods: Individuals with clinical diagnosis of aAP and indication of extraction were selected $(\mathrm{N}=12)$, and biopsies of periapical lesions (PLs) were obtained. For controls, 7 subjects with indication of premolar extraction for orthodontic reasons were selected, and PDL biopsies were obtained. Samples were diagnosed by anatomopathological examination and immunohistochemical staining was carried out to characterize MMPs expression. Results: MMPs-2, $-9,-13$ and -14 detection was limited to PLs and were localized mainly to inflammatory infiltrate on both, PGs and RCs. Additionally, MMP-2 was immunolocalized to fibroblasts from the connective tissue. Conclusions: Whereas MMPs-2, $-9,-13$ and -14 were not detected in healthy periodontal ligament, they were highly expressed on inflammatory infiltrate from PGs and RCs, suggesting a role of these mediators in aAP pathogenesis.
\end{abstract}

Rev. Clin. Periodoncia Implantol. Rehabil. Oral Vol. 4(1); 17-21, 2011.

Key words: Asymptomatic apical periodontitis, periapical lesions, extracellular matrix metalloproteinases.

\section{INTRODUCCIÓN}

La periodontitis apical asintomática (PAa), según la Asociación Americana de Endodoncia(1), se define como la inflamación y destrucción del periodonto apical de origen pulpar, en asociación con un área radiolúcida periapical en ausencia de sintomatología clínica(1).

Junto con la periodontitis, la PAa es considerada como una de las principales causas de pérdida dentaria en adultos $^{(2)}$. En Chile no existen estudios epidemiológicos acerca de esta patología, pero sí se ha reportado una frecuencia de $99.45 \%$ del total de biopsias de lesiones de localización periapical en el Instituto de Referencia de Patología Oral (IREPO) de la Facultad de Odontología de la Universidad de Chile, entre los años 1975 y $2005^{(3)}$.
La PAa se caracteriza por la formación de una lesión osteolítica perirradicular que histológicamente puede corresponder a un granuloma periapical (GPA) o quiste radicular inflamatorio (QRI). Este último se puede generar a partir de la proliferación de los restos epiteliales de Malassez frente al estímulo inflamatorio crónico $^{(4)}$. Se ha descrito que un porcentaje menor al $20 \%$ de las lesiones periapicales aproximadamente corresponderían a un QRI(5).

A pesar de su etiología infecciosa, se considera que las lesiones periapicales (LPAs) se desarrollan principalmente como resultado de la activación de la respuesta inmune frente a la estimulación antigénica continua proveniente de los restos pulpares necróticos en los canales radiculares, producto de la infección bacteriana. Esta respuesta del hospedero involucra una serie de elementos celulares y mediadores moleculares. El infiltrado

1. Cirujano Dentista. Programa de Magíster en Ciencias Odontológicas. Facultad de Odontología, Universidad de Chile. Chile.

2. Profesor Asistente. Área de Endodoncia, Departamento de Odontología Conservadora. Facultad de Odontología, Universidad de Chile. Chile.

3. Profesor Asistente. Área de Periodoncia, Departamento de Odontología Conservadora. Facultad de Odontología, Universidad de Chile. Chile.

4. Profesor Asistente. Area de Patología, Departamento de Patología. Facultad de Odontología, Universidad de Chile. Chile.

5. Laboratorio de Biología Periodontal. Facultad de Odontología, Universidad de Chile. Chile.

Correspondencia autor: Marcela Hernández Ríos. mhernandezrios@gmail.com. Laboratorio de Biología Periodontal, Departamento de Odontología Conservadora, Facultad de Odontología, Universidad de Chile. Chile. Trabajo recibido el 18/01/2011. Aprobado para su publicación el 11/03/2011. Financiamiento: Este trabajo fue financiado por los proyectos DI I 07/02-2 y FONDECYT 1090461 
inflamatorio perirradicular en las LPAs está constituido principalmente por macrófagos, células $\mathrm{T}$ y $\mathrm{B}$, células plasmáticas y polimorfonucleares neutrófilos (PMNs) en cantidades variables ${ }^{(6,7)}$

Entre los mediadores inflamatorios liberados directa e indirectamente por el infiltrado inflamatorio, las metaloproteinasas de matriz extracelular (MMPs), no sólo son capaces de degradar la matriz extracelular (MEC) periodontal, sino que también pueden modificar la actividad biológica de mediadores inflamatorios, tales como citoquinas, quimioquinas y factores de crecimiento, por lo que actuarían como moduladores de los procesos inmunoinflamatorios ${ }^{(8)}$.

El inicio y la progresión de la LPA involucran la degradación y remodelación de la MEC, procesos esenciales para la migración celular y la liberación y activación de factores de crecimiento ${ }^{(9)}$. La acción sinérgica de las MMPs de la familia de las colagenasas (MMPs-1, -8 , -13 y -14) y de las gelatinasas (MMPs-2 y -9) resulta en la hidrólisis del colágeno tipo I, principal componente de los tejidos periodontales. De ellas, las MMPs-13, -14, -2 y -9 corresponden a las principales MMPs expresadas por el tejido óseo durante procesos patológicos osteolíticos $^{(10,11)}$. A nivel óseo, la degradación del colágeno I nativo por las MMPs-13 y -14 es requerida para el acceso del osteoclasto al sitio de reabsorción. Los productos resultantes de la proteólisis del colágeno intersticial realizada por la MMP-13, se desnaturan espontáneamente a temperatura ambiente y se transforman en gelatina, la cual es susceptible a la acción de las MMPs-2 y -9. Además, debido a la activación proteolítica entre ellas, representan una cascada de amplificación que podría prolongar la destrucción tisular ${ }^{(12)}$.

En PAa se ha demostrado la expresión de las MMPs-2, -9 y -13 además de las MMPs-1, -3 y -8 en lesiones periapicales (LPAs) e infiltrado inflamatorio asociado a estas lesiones ${ }^{(10,13-18)}$, no así en ligamento periodontal sano, mientras que no existen estudios previos referentes a la expresión de MMP-14 en estas lesiones.

Adicionalmente, resultados previos obtenidos por nuestro grupo de trabajo han demostrado la presencia y/o niveles elevados de MMPs-2 y -9 en fluido gingival crevicular de dientes con PAa en relación con fluido gingival crevicular de dientes sanos ${ }^{(10)}$.

Debido a los antecedentes expuestos anteriormente, el objetivo de este estudio fue determinar el patrón de expresión de las MMPs-2, $-9,-13$ y -14 en granulomas periapicales (GPAs), quistes radiculares inflamatorios (QRIs) y ligamento periodontal sano (LS).

\section{MATERIALES Y MÉTODOS}

El estudio realizado es de carácter analítico transversal y cuenta con la aprobación del Comité de Ética de la Facultad de Odontología de la Universidad de Chile.

\section{Selección de Sujetos.}

Se incluyeron pacientes que acudieron a la Clínica de Cirugía de la Facultad de Odontología de la Universidad de Chile durante el año 2008. Se seleccionaron 12 sujetos con diagnóstico clínico de PAa e indicación de exodoncia del diente afectado. El diagnóstico se realizó mediante anamnesis, examen clínico y examen radiológico y los datos se consignaron en una ficha clínica. Los pacientes fueron informados del procedimiento a realizar y luego se les solicitó la firma de un consentimiento informado aprobado por el Comité de Ética de la Facultad de Odontología de la Universidad de Chile. Se incluyeron en el presente estudio pacientes que presentaran dientes con indicación de exodoncia que no respondieran a los test de diagnóstico pulpar (frío probado con cloruro de etilo, calor probado con transpoliisopreno), con respuesta normal o levemente aumentada a la percusión y destrucción ósea apical $>5 \mathrm{~mm}$ en relación al diente en cuestión observada al examen radiográfico (imagen radiográfica compatible con GPA O QRI). Se excluyeron del presente estudio individuos que presentaran tratamientos con antibioterapia, corticoides o antiinflamatorios en los últimos 6 meses, enfermedades sistémicas y/o embarazo. Como controles se seleccionaron 7 sujetos sanos, con indicación ortodóncica de exodoncia de premolares.

\section{Obtención y Procesamiento de las Muestras.}

Luego de realizadas las exodoncias, se obtuvieron las biopsias periapicales, se lavaron con suero fisiológico y fijaron en formalina tamponada al $10 \%$ a $\mathrm{pH} 7.4$. Las muestras que contenían tejidos duros fueron descalcificadas en ácido etilendiaminotetraacético (EDTA) al $5 \%$. Las muestras fueron posteriormente embebidas en parafina y se realizó el procesamiento de rutina, tinción hematoxilina-eosina $(\mathrm{H}-\mathrm{E})$ y el diagnóstico anátomopatológico de las muestras, como QRI, GPA o LS.

\section{Inmunohistoquímica.}

Para efectuar la detección de los mediadores en estudio, se efectuaron tinciones inmunohistoquímicas. Se obtuvieron cortes de $6 \mu \mathrm{m}$ de GPA, QRI y LS. Una vez desparafinados, se bloqueó la peroxidasa endógena con peróxido de hidrógeno/metanol al $3 \%$ por $10 \mathrm{~min}$, se lavaron con tampón fosfato salino (PBS Hemagen Diagnostics INC $\circledast$, MA, USA) se realizó el desenmascaramiento del antígeno con Proteinasa $\mathrm{K}$ según indicaciones del fabricante (Novocastra ${ }^{\circ}$, Lab. Novo, Newcastle, UK). Se repitió el lavado con PBS y se bloquearon los sitios inespecíficos con suero equino $2.5 \%$ por 10 minutos (Kit $A B C$ Universal, RTU Vectastain $®$ Kit for laboratory use, Burlingame, CA, USA). Se agregó el anticuerpo primario monoclonal correspondiente: anti MMP-2, MMP-9, MMP-13 o MMP-14 (R\&D Systems, Inc $®$, Minneapolis, MN, USA), en diluciones de 1:5000, 1:1000, 1:20 y 1:100, respectivamente, en solución de suero equino al $2.5 \%$. Los cortes se dejaron incubando en una cámara húmeda a $4^{\circ} \mathrm{C}$ durante toda la noche. Los controles negativos fueron incubados con suero equino $2.5 \%$ sin el anticuerpo primario. Al día siguiente, las muestras se lavaron con PBS y la reacción se visualizó mediante el kit ABC (Kit ABC Universal, RTU Vectastain ${ }^{\circledR}$ Kit, Burlingame, CA, USA) según instrucciones del fabricante. Tras volver a lavar con PBS, se reveló con DAB Kit (Peroxidasa Sustrate Kit DAB SK4100, Zymed Labs INC®, San Francisco, CA, USA) y se realizó la contratinción con hematoxilina. Finalmente, las muestras fueron deshidratadas y montadas con medio de montaje hidrofóbico (Flotex®, Lerner Laboratories, Pittsburgh, PA, USA). Los cortes estudiados se examinaron con un microscopio óptico (Zeiss, Axiostar Plus $\AA_{,} N Y$, USA) y se fotografiaron las áreas más representativas de cada espécimen con una cámara de fotos digital (Canon, Powershot A640, Tokio, Japón).

\section{RESULTADOS}

Las características clínicas y diagnósticos respectivos de los sujetos en estudio se presentan en la Tabla 1. De las 19 muestras obtenidas, siete fueron diagnosticadas histopatologicamente como GPA cinco como QRI y siete como LS. No se observó presencia de epitelio en ninguno de los GPA estudiados.

Las MMPs-2, $-9,-13$ y -14 se detectaron en todas las muestras de GPAs y QRIs mientras que no se detectaron en las muestras de LS. En general, todas las MMPs estudiadas se inmunolocalizaron principalmente en el infiltrado inflamatorio de las LPAs.

Con respecto a la MMP-2, tanto en GPA como QRI, se observó inmunopositividad en el infiltrado inflamatorio, particularmente en plasmocitos, linfocitos y en células endoteliales. Además se observaron fibroblastos/fibrocitos inmunopositivos en la cápsula quística (Figura 1).

Tabla 1. Cuadro resumen de características de los sujetos del estudio.

\begin{tabular}{|c|c|c|c|}
\hline \hline Diagnóstico & Edad (Promedio \pm DS) & Mujeres & $\mathbf{n}$ \\
\hline GPA & $48.5 \pm 17.1$ & 2 & 7 \\
\hline QRI & $52.6 \pm 1.5$ & 2 & 5 \\
\hline Ligamento sano & $14.7 \pm 3.09$ & 6 & 7 \\
\hline
\end{tabular}

Tabla 2. Cuadro comparativo de la expresión de MMP-2, -9, -13 y -14 en diferentes tipos celulares presentes en GPA y QRI

\begin{tabular}{|c|c|c|c|c|c|c|c|c|}
\hline \hline & \multicolumn{2}{|c|}{ MMP-2 } & \multicolumn{2}{c|}{ MMP-9 } & \multicolumn{2}{c|}{ MMP-13 } & \multicolumn{2}{c|}{ MMP-14 } \\
\hline Morfología celular & GPA & QRI & GPA & QRI & GPA & QRI & GPA & QRI \\
\hline PMN & - & - & + & + & - & - & - & - \\
\hline Plasmocitos & + & + & + & + & + & + & + & + \\
\hline Linfocitos & + & + & + & + & - & - & - & - \\
\hline Células tipo macrófago & - & - & - & - & - & + & - & + \\
\hline Células endoteliales & + & + & + & - & - & - & + & + \\
\hline Fibroblastos & + & + & - & - & - & - & - & - \\
\hline Queratinocitos & - & - & - & + & - & - & - & + \\
\hline
\end{tabular}




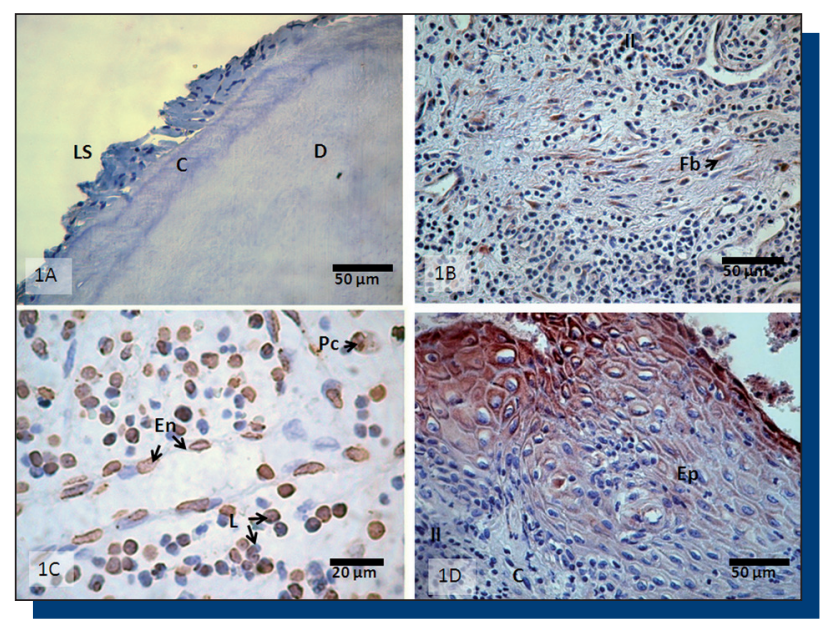

Figura 1. Inmunodetección de MMP-2 en LS (A), GPA (B, C), QRI (D). Las flechas indican células inmunoreactivas. LS: Ligamento sano; C: Cemento; D: Dentina; Fb: Fibroblasto; En: Célula endotelial; Pc: Plasmocitos; L: Linfocitos; Ep: Epitelio quístico; C: Cápsula quística; II: Infiltrado inflamatorio.

La MMP-9, de modo similar a la MMP-2, se inmunolocalizó en el infiltrado inflamatorio de GPA y QRI, particularmente en PMN, linfocitos y plasmocitos. En QRI, se identificó en el epitelio quístico, y en GPA, en células endoteliales (Figura 2).

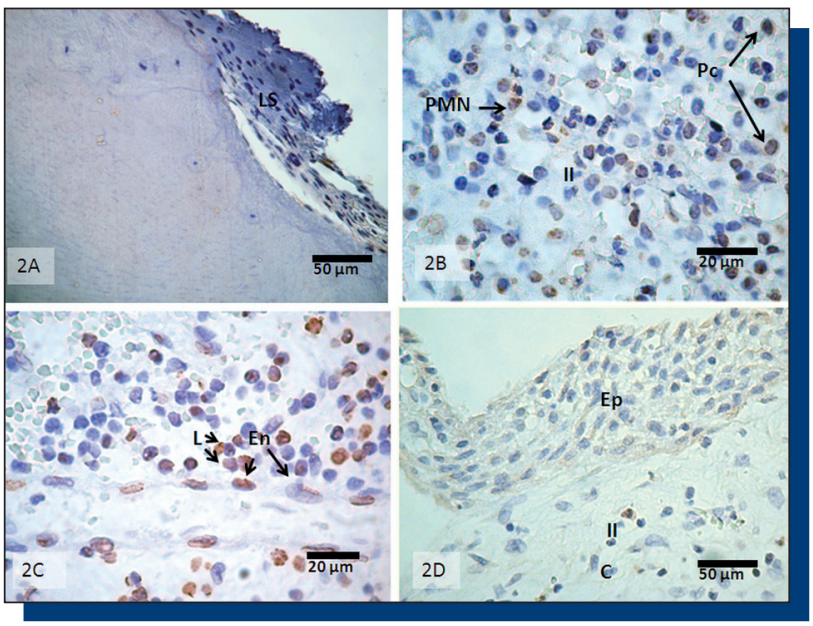

Figura 2. Inmunodetección de MMP-9 en LS (A), GPA (B, C), QRI (D). Las flechas indican células inmunoreactivas. LS: Ligamento sano; PMN: Polimorfonuclear neutrófilo; Pc: Plasmocito; En: Célula endotelial; L: Linfocitos; Ep: Epitelio quístico; C: Cápsula quística; II: Infiltrado inflamatorio.

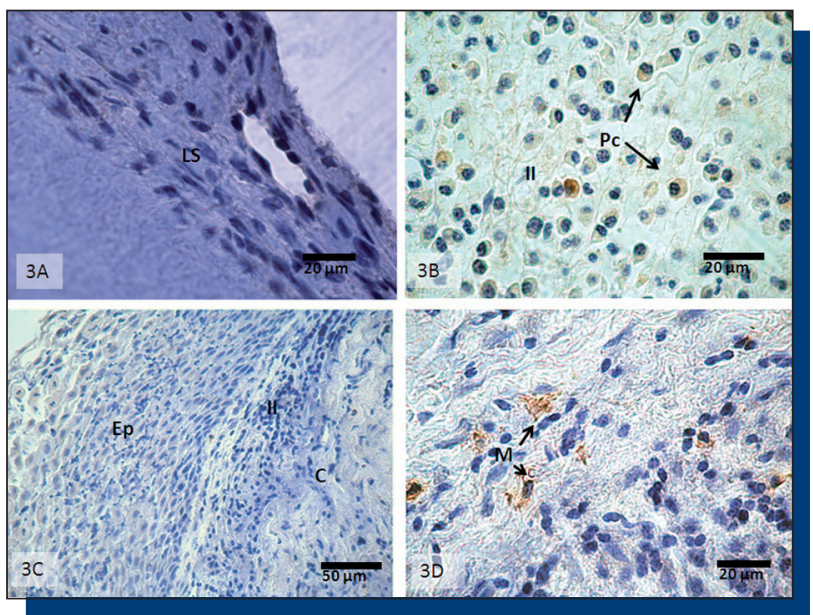

Figura 3. Inmunodetección de MMP-13 en LS (A), GPA (B), QRI (C, D). Las flechas indican células inmunoreactivas. LS: Ligamento sano; II: Infiltrado inflamatorio; Pc: Plasmocito; Ep: Epitelio quístico; C: Cápsula quística; M: Célula tipo-macrófago.
La MMP-13 se inmunolocalizó en el infiltrado inflamatorio de GPA y QRI, principalmente en plasmocitos. En QRI además se identificó la expresión de MMP-13 en células con morfología compatible con macrófagos (Figura 3).

Por último, la inmunodetección de MMP-14 se localizó en GPA y QRI a nivel del infiltrado inflamatorio, especialmente en plasmocitos, y en células endoteliales. En QRI se observaron células inmunopositivas en el epitelio, cápsula, principalmente a nivel del infiltrado inflamatorio, de manera particular en células con morfología compatible con macrófagos (Figura 4).
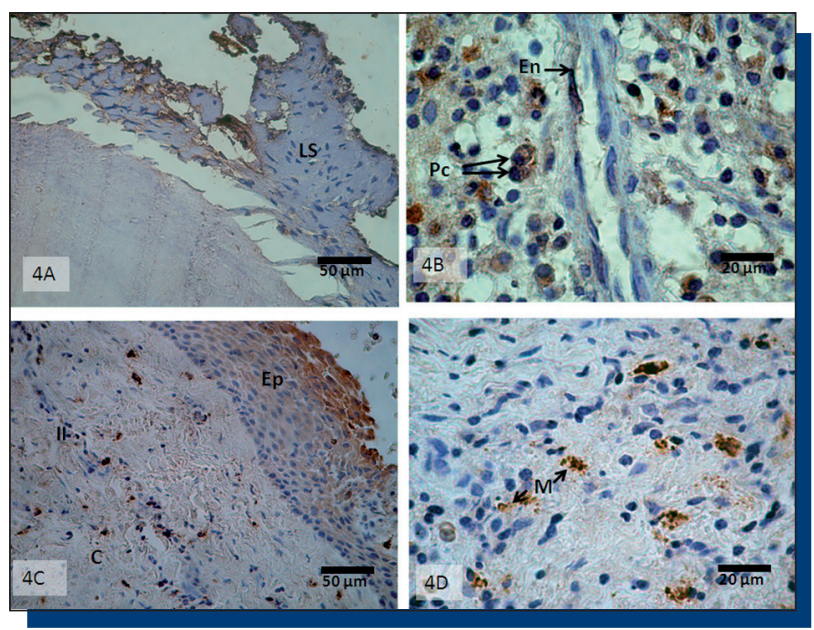

Figura 4. Inmunodetección de MMP-14 en LS (A), GPA(B), QRI (C, D). Las flechas indican células inmunoreactivas. LS: Ligamento sano; Pc: Plasmocito; En: Célula endotelial; Ep: Epitelio quístico; C: Cápsula quística; II: Infiltrado inflamatorio; M: Célula tipo-macrófago.

\section{DISCUSIÓN}

Las LPAs constituyen un mecanismo de respuesta del hospedero frente a la infección bacteriana del sistema de canales radiculares del diente ${ }^{(7)}$. Esta respuesta involucra la presencia de células inmunes e inflamatorias, así como de mediadores inflamatorios, que incluyen a las MMPs y que podrían estar participando en la etiopatogenia de estas lesiones. En este estudio se demostró la presencia de las MMPs-2, -9, -13 y -14 en GPA y QRI, pero no en LS, sustentando la propuesta de que estas MMPs se asocian con la formación y/o progresión de los GPAs y QRIs.

La patogénesis de la periodontitis apical involucra la degradación de diversos componentes de la MEC como resultado de la infección bacteriana dentro del sistema de canales radiculares ${ }^{(7)}$. Las MMPs pertenecientes a las familias de las colagenasas y gelatinasas, tendrían un rol preponderante en el inicio y/o progresión de las LPAs al degradar directamente el colágeno constituyente de los tejidos blandos y óseos del periodonto(19-21). Además, las MMPs no sólo son capaces de degradar la MEC periodontal, sino que mediante proteólisis limitada pueden modificar la actividad biológica de mediadores inflamatorios, actuando como moduladores de los procesos inmunoinflamatorios ${ }^{(8)}$

Los resultados obtenidos en este estudio respecto a la presencia de MMP-2 en LPAs, concuerdan con estudios anteriores ${ }^{(14,22)}$, donde se determinó la expresión de MMP-2 en plasmocitos, linfocitos y células endoteliales, tanto en GPA como QRI.

Se ha descrito que la MMP-2, junto con la MMP-9, participa en el proceso de reabsorción ósea en la remoción de la matriz ósea orgánica. Las MMPs-2 y -9 degradan gelatina, que corresponde al colágeno desnaturalizado(9), producto de la acción previa de las MMPs con actividad colagenasa (MMPs-1, -8, -13 y -14). Además, la forma activa de la MMP-2 se ha detectado previamente en el fluido gingival crevicular (FGC) de dientes con PAa pero no en FGC de dientes sanos. Los autores sugieren que la MMP-2 podría representar un marcador útil para el monitoreo de $\mathrm{PAa}$ en el FGC(10). Consecuentemente, la MMP-2 podría estar actuando en la degradación de la MEC, favoreciendo la expansión de la lesión y, junto con algunas citoquinas, como lo son TNF- $\alpha$, IL- $1 \alpha$, IL- $1 \beta$, y el sistema RANKL/RANK/OPG ${ }^{(23)}$, podría contribuir también a la destrucción ósea ${ }^{(22)}$.

En relación a la MMP-9, su detección fue similar a estudios anteriores, donde también se describió su expresión en PMN, linfocitos, plasmocitos, células endoteliales y epiteliales ${ }^{(16,22,24)}$. La MMP-9 actuaría sobre el reclutamiento de preosteoclastos al tejido óseo y su migración 
desde la médula ósea para la diferenciación osteoclástica y reabsorción ósea ${ }^{(25)}$. Se ha reportado que MMP-9 es una enzima cooperativa que participa en el ciclo de activación-reabsorción y formación involucrado en la remodelación y destrucción patológica de hueso ${ }^{(13)}$ y se ha asociado con la destrucción de tejido periodontal en otras condiciones como periodontitis crónica y periimplantitis ${ }^{(26,27)}$. De modo similar a la MMP-2, se han descrito niveles de proMMP-9 aumentados en FGC de dientes con PAa en comparación con FGC de dientes sanos, de modo que también podría representar un marcador útil para el monitoreo de $\mathrm{PAa}^{(10)}$.

Con respecto a la expresión de MMP-13, los resultados de presente estudio concuerdan con estudios anteriores ${ }^{(13,15)}$. Se obtuvo inmunopositividad en plasmocitos tanto en GPAs como en QRIs. La expresión de MMP-13 por estas células se ha asociado a un fenotipo particularmente destructivo, ya que la presencia de esta colagenasa se ha observado también en numerosas lesiones osteolíticas de diversa naturaleza, tales como quistes odontogénicos y plasmocitomas malignos ${ }^{(13)}$. Por otro lado, MMP-13 se ha detectado fuertemente en lesiones periapicales epitelizadas en humanos, donde pareciera contribuir a la migración de células epiteliales e invasión de tejido de granulación durante la conversión de un GPA con epitelio a un QRI(15). De modo similar, se ha observado la expresión de MMP-13 en encía de pacientes con periodontitis crónica, donde podría tener un rol en el crecimiento proliferativo del epitelio dentro del tejido conectivo ${ }^{(28)}$. Sin embargo, en este estudio no observamos una expresión consistente de la MMP-13 en el revestimiento epitelial de QRIs, aunque este hecho podría explicarse por un estado más bien quiescente de los epitelios quísticos estudiados. Además, las MMPs-13, -9 y catepsina K producidas por osteoclastos, permitirían la remoción de la matriz orgánica durante la reabsorción ósea ${ }^{(29,30)}$. Se ha propuesto, además, que entre los mecanismos moleculares de la expansión de los QRI, estaría involucrado un desbalance entre MMPs y sus inhibidores tisulares (TIMPs)(15).

Se sabe que la actividad de MMP-13 en extractos de tejido gingival y FGC de sitios con periodontitis crónica está patológicamente elevada comparada con el tejido periodontal y FGC de dientes sanos ${ }^{(25,31)}$. La MMP-13 fue descrita originalmente en cáncer de mama ${ }^{(32)}$, yse expresa en carcinomas de células escamosas de cabeza y cuello donde su expresión se correlaciona con su capacidad invasora ${ }^{(33)}$. La MMP-13 también ha sido descrita en tumores odontogénicos y su expresión se ha relacionado con el comportamiento biológico de estas lesiones ${ }^{(34)}$. En artritis reumatoide y osteoartritis se considera como la enzima responsable de la reabsorción ósea y destrucción de cartílago ${ }^{(35)}$. Finalmente, la actividad de la MMP-13 podría iniciar la reabsorción ósea; en soporte de lo anterior, en un estudio realizado en periodontitis crónica progresiva se encontró aumento de la actividad de la MMP-13 en sitios con progresión en asociación con niveles de ICTP, marcador de catabolismo óseo ${ }^{(36)}$.

Nuestros resultados demuestran por primera vez la presencia de MMP-14 en LPAs. MMP-14 se ha descrito como una de las enzimas proteolíticas responsables de la degradación de la matriz orgánica ósea producida por células como osteoblastos y osteoclastos ${ }^{(37,38)}$. Además, por la participación que tiene en la activación de proMMP-2 junto con TIMP- $2^{(39)}$ y proMMP-13, tendría un rol indirecto en la degradación de la MEC necesaria para la reabsorción ósea característica de las LPAs.

En el presente estudio se observó la presencia de MMP-13 y -14 en células con morfología compatible con macrófagos en QRIs. Estas células tienen un rol fundamental en la patogenia de las LPAs, ya que representan la primera fuente de citoquinas, enzimas y otros mediadores con efecto proinflamatorio y osteolítico en PAa, tales como $\mathrm{IL}-1 \alpha$, IL-1 $\beta$ y TNF- $\alpha$, MMPs y PGs ${ }^{(40)}$. Éstos podrían participar tanto en la respuesta protectora como en la formación y perpetuación de la $P A a^{(41,42)}$. También se ha postulado que las citoquinas y quimioquinas derivadas de los macrófagos podrían estar implicadas en la proliferación de restos epiteliales de Malassez durante la formación de un $\mathrm{QRI}(41,43)$.

Además de la destrucción directa de la matriz extracelular periodontal, han sido descritos sustratos bioactivos in vivo para estas enzimas. De este modo, la MMP-13 puede activar a la proMMP-9 y proMMP-13 y este mecanismo se ha demostrado en la patogenia de la periodontitis crónica ${ }^{(12,39,44,45)}$. Adicionalmente, se ha descrito que MMP-9 activa a proMMP-13 y proMMP-2 in vitro ${ }^{(39,46)}$, generando una cascada de amplificación que podría estar prolongando la destrucción tisular ${ }^{(12)}$. En un estudio reciente, se demostró en GPAs que las MMPs que se expresaban de mayor manera son las MMPs-2, -9 y $-13^{(24)}$.

Si bien en éste y otros estudios se observa expresión de MMPs en PAa, la función de estas enzimas en la patogenia de esta enfermedad aun no se conoce claramente. Se requieren nuevas investigaciones para establecer el rol de ellas y su interacción con los demás mediadores inflamatorios presentes. Recientemente, se ha descrito que las MMPs podrían actuar de manera protectora, inhibiendo la progresión de la destrucción de tejido periapical inducido por bacterias, como se ha reportado previamente en periodontitis crónica y apical ${ }^{(47)}$. Se ha observado que, en inflamación pulpar y periapical, contrariamente a lo esperado, la inhibición general de la actividad de las MMPs aumenta la propagación de la necrosis pulpar y la tasa de crecimiento de las LPAs, indicando que las MMPs pueden tener propiedades antiinfecciosas y/o antiinflamatorias desconocidas anteriormente ${ }^{(47)}$. De este modo, las MMPs forman redes altamente complejas. Dentro de la gran familia de las MMPs, no se puede descartar que algunas de ellas puedan tener roles antagónicos. Adicionalmente, estudios de periodontitis experimental evidenciaron una mayor pérdida ósea en ratones MMP-8-/- en comparación con los tipos silvestres en periodontitis experimental inducida por $\mathrm{P}$. gingivalis y por LPS conjuntamente con una reducción de la quimioquina para PMNs LIX(48-50). Estos antecedentes sugieren que MMPs como la MMP-8 podrían desempeñar un papel protector en concentraciones fisiológicas, mientras que sus niveles y/o actividad elevados patológicamente podrían tener, por el contrario, un efecto destructivo sobre los tejidos periodontales.

En síntesis, la presencia de las MMPs-2, -9, -13y-14 se encuentra íntimamente relacionada entre sí y con los mediadores inflamatorios osteolíticos presentes en la PAa. Un estudio más acabado de estas MMPs podría conducir al desarrollo de métodos moleculares complementarios al diagnóstico y monitoreo clínico, marcadores de pronóstico y nuevos blancos de terapia farmacológica que conduzcan a una mejora futura de los actuales enfoques clínicos.

\section{CONCLUSIÓN}

Las MMPs-2, -9, -13 y -14 se expresan en diversos tipos celulares presentes en GPAs y QRIs, y no así en LS. Por esta razón, estas MMPs podrían estar asociadas con la formación y/o progresión de los GPAs y QRIs.

\section{REFERENCIAS BIBLIOGRÁFICAS}

1. Glickman GN. AAE Consensus Conference on Diagnostic Terminology: background and perspectives. J Endod, 2009 Dec; 35(12): 1619-1620. 2. Takahashi K. Microbiological, pathological, inflammatory, immunological and molecular biological aspects of periradicular disease. Int Endod J, 1998 Sep; 31(5): 311-325.

3. Ortega A, Fariña V, Gallardo A, Espinoza I, Acosta S. Nonendodontic periapical lesions: A retrospective study in Chile. Int Endod J, 2007 May; 40(5): 386-390.

4. Liapatas S, Nakou M, Rontogianni D. Inflammatory infiltrate of chronic periradicular lesions: An immunohistochemical study. Int Endod J, 2003 Jul; 36(7): 464-471.

5. Ramachandran Nair PN, Pajarola G, Schroeder HE. Types and incidence of human periapical lesions obtained with extracted teeth. Oral Surg Oral Med Oral Pathol Oral Radiol Endod, 1996 Jan; 81(1): 93-102.
6. Matsuo T, Ebisu S, Shimabukuro Y, Ohtake T, Okada H. Quantitative analysis of immunocompetent cells in human periapical lesions: correlations with clinical findings of the involved teeth. J Endod, 1992 Oct; 18(10): 497-500.

7. Nair PN. Pathogenesis of apical periodontitis and the causes of endodontic failures. Crit Rev Oral Biol Med, 2004; 15(6): 348-381.

8. Parks WC, Wilson CL, Lopez-Boado YS. Matrix metalloproteinases as modulators of inflammation and innate immunity. Nat Rev Immunol, 2004 Aug; 4(8): 617-629.

9. Visse R, Nagase H. Matrix metalloproteinases and tissue inhibitors of metalloproteinases: Structure, function, and biochemistry. Circ Res, 2003 May 2; 92(8): 827-839.

10. Belmar MJ, Pabst C, Martínez B, Hernández M. Gelatinolytic activity in gingival crevicular fluid from teeth with periapical lesions. Oral Surg Oral Med Oral Pathol Oral Radiol Endod, 2008 Jun; 105(6): 801-806. 
11. Hernández M, Gamonal J, Tervahartiala T, Mantyla P, Rivera $O$, Dezerega A et al. Associations between matrix metalloproteinase-8 and -14 and myeloperoxidase in gingival crevicular fluid from subjects with progressive chronic periodontitis: A longitudinal study. J Periodontol, 2010 Nov; 81(11): 1644-1652.

12. Hernández Ríos M, Sorsa T, Obregon F, Tervahartiala T, Valenzuela MA, Pozo $\mathrm{P}$ et al. Proteolytic roles of matrix metalloproteinase (MMP)-13 during progression of chronic periodontitis: Initial evidence for MMP-13/MMP-9 activation cascade. J Clin Periodontol, 2009 Dec; 36(12): 1011-1017.

13. Wahlgren J, Maisi P, Sorsa T, Sutinen M, Tervahartiala T, Pirila E et al. Expression and induction of collagenases (MMP-8 and -13) in plasma cells associated with bone-destructive lesions. J Pathol, 2001 Jun; 194(2): 217-224

14. Shin SJ, Lee JI, Baek SH, Lim SS. Tissue levels of matrix metalloproteinases in pulps and periapical lesions. J Endod, $2002 \mathrm{Apr}$; 28(4): 313-315.

15. Leonardi R, Lanteri E, Stivala F, Travali S. Immunolocalization of CD44 adhesion molecules in human periradicular lesions. Oral Surg Oral Med Oral Pathol Oral Radiol Endod, 2000 Apr; 89(4): 480-485.

16. Carneiro E, Menezes R, Garlet GP, Garcia RB, Bramante CM, Figueira $\mathrm{R}$ et al. Expression analysis of matrix metalloproteinase- 9 in epithelialized and nonepithelialized apical periodontitis lesions. Oral Surg Oral Med Oral Pathol Oral Radiol Endod, 2009 Jan; 107(1): 127-132.

17. Buzoglu HD, Unal H, Ulger C, Mert S, Kucukyildirim S, Er N. The zymographic evaluation of gelatinase (MMP-2 and -9) levels in acute and chronic periapical abscesses. Oral Surg Oral Med Oral Pathol Oral Radiol Endod, 2009 Nov; 108(5): 121-126.

18. Paula-Silva FW, da Silva LA, Kapila YL. Matrix metalloproteinase expression in teeth with apical periodontitis is differentially modulated by the modality of root canal treatment. J Endod, $2010 \mathrm{Feb}$; 36(2): 231-237. 19. Hill PA, Murphy G, Docherty AJ, Hembry RM, Millican TA, Reynolds $\mathrm{JJ}$ et al. The effects of selective inhibitors of matrix metalloproteinases (MMPs) on bone resorption and the identification of MMPs and TIMP-1 in isolated osteoclasts. J Cell Sci, 1994 Nov; 107 (Pt 11): 3055-3064.

20. Hill PA, Docherty AJ, Bottomley KM, O'Connell JP, Morphy JR, Reynolds JJ et al. Inhibition of bone resorption in vitro by selective inhibitors of gelatinase and collagenase. Biochem J, 1995 May 15; 308 (Pt 1): 167-175.

21. Golub LM, Lee HM, Ryan ME, Giannobile WV, Payne J, Sorsa T. Tetracyclines inhibit connective tissue breakdown by multiple nonantimicrobial mechanisms. Adv Dent Res, 1998 Nov; 12(2): 12-26.

22. Corotti MV, Zambuzzi WF, Paiva KB, Menezes R, Pinto LC, Lara VS et al. Immunolocalization of matrix metalloproteinases -2 and -9 during apical periodontitis development. Arch Oral Biol, 2009 Aug; 54(8): 764-771.

23. Kawashima N, Suzuki N, Yang G, Ohi C, Okuhara S, NakanoKawanishi $\mathrm{H}$ et al. Kinetics of RANKL, RANK and OPG expressions in experimentally induced rat periapical lesions. Oral Surg Oral Med Oral Pathol Oral Radiol Endod, 2007 May; 103(5): 707-711.

24. de Paula-Silva FW, D'Silva NJ, da Silva LA, Kapila YL. High matrix metalloproteinase activity is a hallmark of periapical granulomas. J Endod, 2009 Sep; 35(9): 1234-1242.

25. Hernández M, Martínez B, Tejerina JM, Valenzuela MA, Gamonal J. MMP-13 and TIMP-1 determinations in progressive chronic periodontitis. J Clin Periodontol, 2007 Sep; 34(9): 729-735

26. Ingman T, Tervahartiala T, Ding Y, Tschesche H, Haerian A, Kinane DF et al. Matrix metalloproteinases and their inhibitors in gingival crevicular fluid and saliva of periodontitis patients. J Clin Periodontol, 1996 Dec; 23(12): 1127-1132.

27. Puente XS, Sanchez LM, Overall CM, Lopez-Otin C. Human and mouse proteases: A comparative genomic approach. Nat Rev Genet, 2003 Jul; 4(7): 544-558

28. Uitto VJ, Airola K, Vaalamo M, Johansson N, Putnins EE, Firth JD et al. Collagenase-3 (matrix metalloproteinase-13) expression is induced in oral mucosal epithelium during chronic inflammation. Am J Pathol, 1998 Jun; 152(6): 1489-1499.

29. Teitelbaum SL. Bone resorption by osteoclasts. Science, 2000 Sep 1; 289(5484): 1504-1508.

30. Teitelbaum SL. Osteoclasts: What do they do and how do they do it? Am J Pathol, 2007 Feb; 170(2): 427-435.

31. Ilgenli T, Vardar-Sengul S, Gurkan A, Sorsa T, Stackelberg S, Kose $\mathrm{T}$ et al. Gingival crevicular fluid matrix metalloproteinase-13 levels and molecular forms in various types of periodontal diseases. Oral Dis, 2006 Nov; 12(6): 573-579.
32. Freije JM, Diez-Itza I, Balbin M, Sanchez LM, Blasco R, Tolivia J et al. Molecular cloning and expression of collagenase-3, a novel human matrix metalloproteinase produced by breast carcinomas. J Biol Chem, 1994 Jun 17; 269(24): 16766-16773.

33. Ala-aho R, Ahonen M, George SJ, Heikkila J, Grenman R, Kallajoki M et al. Targeted inhibition of human collagenase-3 (MMP-13) expression inhibits squamous cell carcinoma growth in vivo. Oncogene, 2004 Jul 1; 23(30): 5111-5123.

34. Wahlgren J, Vaananen A, Teronen O, Sorsa T, Pirila E, Hietanen J et al. Laminin-5 gamma 2 chain is colocalized with gelatinase-A (MMP-2) and collagenase-3 (MMP-13) in odontogenic keratocysts. J Oral Pathol Med, 2003 Feb; 32(2): 100-107.

35. Martel-Pelletier J, Pelletier JP. Wanted the collagenase responsible for the destruction of the collagen network in human cartilage! $\mathrm{Br} J$ Rheumatol, 1996 Sep; 35(9): 818-820.

36. Hernández M, Valenzuela MA, López-Otin C, Álvarez J, López $\mathrm{JM}$, Vernal $\mathrm{R}$ et al. Matrix metalloproteinase -13 is highly expressed in destructive periodontal disease activity. J Periodontol, 2006 Nov; 77(11): 1863-1870.

37. Mansell JP, Tarlton JF, Bailey AJ. Expression of gelatinases within the trabecular bone compartment of ovariectomized and parathyroidectomized adult female rats. Bone, 1997 Jun; 20(6): 533-538.

38. Greenwald RA, Golub LM, Ramamurthy NS, Chowdhury M, Moak SA, Sorsa T. In vitro sensitivity of the three mammalian collagenases to tetracycline inhibition: Relationship to bone and cartilage degradation. Bone, 1998 Jan; 22(1): 33-38.

39. Folgueras AR, Pendas AM, Sanchez LM, Lopez-Otin C. Matrix metalloproteinases in cancer: From new functions to improved inhibition strategies. Int J Dev Biol, 2004; 48(5-6): 411-424.

40. Metzger Z. Macrophages in periapical lesions. Endod Dent Traumatol, 2000 Feb; 16(1): 1-8.

41. Marton IJ, Kiss C. Protective and destructive immune reactions in apical periodontitis. Oral Microbiol Immunol, 2000 Jun; 15(3): 139-150.

42. Vernal R, Dezerega A, Dutzan N, Chaparro A, Leon R, Chandia S et al. RANKL in human periapical granuloma: possible involvement in periapical bone destruction. Oral Dis, 2006 May; 12(3): 283-289.

43. Silva TA, Garlet GP, Lara VS, Martins W, Jr., Silva JS, Cunha FQ. Differential expression of chemokines and chemokine receptors in inflammatory periapical diseases. Oral Microbiol Immunol, 2005 Oct; 20(5): 310-316.

44. Knauper V, Cowell S, Smith B, López-Otin C, O'Shea M, Morris H et al. The role of the C-terminal domain of human collagenase-3 (MMP13 ) in the activation of procollagenase- 3 , substrate specificity, and tissue inhibitor of metalloproteinase interaction. J Biol Chem, 1997 Mar 21; 272(12): 7608-7616.

45. Knauper V, Will H, López-Otin C, Smith B, Atkinson SJ, Stanton $\mathrm{H}$ et al. Cellular mechanisms for human procollagenase-3 (MMP-13) activation. Evidence that MT1-MMP (MMP-14) and gelatinase a (MMP-2) are able to generate active enzyme. J Biol Chem, 1996 Jul 19; 271(29): 17124-17131.

46. Overall CM. Molecular determinants of metalloproteinase substrate specificity: Matrix metalloproteinase substrate binding domains, modules, and exosites. Mol Biotechnol, 2002 Sep; 22(1): 51-86.

47. Tjaderhane L, Hotakainen T, Kinnunen S, Ahonen M, Salo T. The effect of chemical inhibition of matrix metalloproteinases on the size of experimentally induced apical periodontitis. Int Endod J, 2007 Apr; 40(4): 282-289.

48. Owen CA, Hu Z, Lopez-Otin C, Shapiro SD. Membrane-bound matrix metalloproteinase-8 on activated polymorphonuclear cells is a potent tissue inhibitor of metalloproteinase-resistant collagenase and serpinase. J Immunol, 2004 Jun 15; 172(12): 7791-7803.

49. Kuula H, Salo T, Pirila E, Tuomainen AM, Jauhiainen M, Uitto VJ et al. Local and systemic responses in matrix metalloproteinase 8-deficient mice during Porphyromonas gingivalis-induced periodontitis. Infect Immun, 2009 Feb; 77(2): 850-859.

50. Hernández M, Gamonal J, Salo T, Tervahartiala T, Hukkanen M, Tjaderhane $L$ et al. Reduced expression of lipopolysaccharide-induced CXC chemokine in Porphyromonas gingivalis-induced experimental periodontitis in matrix metalloproteinase-8 null mice. J Periodontal Res, 2011 Feb; 46(1): 58-66. 